\title{
Editorial
}

\section{Hematocrit and Prohepcidin: Causation or Simply Association?}

\author{
Francesco Locatelli Pietro Pozzoni \\ Department of Nephrology and Dialysis, A. Manzoni Hospital, Lecco, Italy
}

Starting from the observation that patients with chronic hemodialysis (HD) and chronic anemia may share the same laboratory findings (i.e. low transferrin saturation and high ferritin levels), Hsu et al. [1] investigated the relationship between plasma levels of prohepcidin, the inactive prohormone of hepcidin, and various indices of the iron status, inflammation and erythropoietin treatment in 71 prevalent HD patients. Since hepcidin, a liver-derived peptide stimulated by inflammatory cytokines [2], acts as a negative regulator of intestinal iron absorption and iron release from macrophages, it is believed to represent the molecular link between chronic inflammation and anemia, and may also play a role in anemic patients with high ferritin levels on chronic HD. The study failed to demonstrate any relationship between indices of the iron status and serum prohepcidin concentrations in the HD patients, but hematocrit levels proved to be independently and positively correlated with plasma prohepcidin levels also at multivariate analysis. Based on these results, the authors suggested that hematocrit levels may have a beneficial effect on the regulation of prohepcidin production, i.e. they postulated a causal link between hematocrit and prohepcidin levels. The results of the study are intriguing, because they offer the first evidence of a relationship between hepcidin expression and the degree of anemia in the setting of end-stage renal disease. However, the conclusions drawn by the authors require some caution. The study was indeed designed as a cross-sectional study, known to highlight the existence of associations but unable to demonstrate cause-effect relationships. Although support was provided by the results of previous experimental studies [3], a cross-sectional correlation is not sufficient to indicate whether hematocrit stimulates hepcidin production by the liver: in the absence of the time factor, it is unfortunately impossible to speculate which comes 'first' and which 'afterwards'. For the same reason, it is not surprising that no relationship was found between prohepcidin and ferritin levels, since the cross-sectional design of the study could not avoid the potentially confounding effect of iron supplementation, which is known to be one of the factors stimulating hepcidin expression. As the patients with the lowest ferritin levels are probably those receiving the highest iron dose, the cross-sectional design may have masked possible positive correlations between hepcidin, ferritin and iron supplements, thus leading to the erroneous conclusion that they were not interrelated. In future trials, possible correlations between hepcidin and ferritin should be studied in patients not receiving iron supplementation. In conclusion, the results of this preliminary study by Hsu et al. [1] deserve particular attention because they present the first results of a clear and direct relationship between plasma prohepcidin and hematocrit levels in chronic HD patients. However, these findings require further evaluation in order to clarify whether the altered hepcidin expression is pathogenetically related to renal anemia or simply an epiphenomenon. Longitudinal cohort studies in which prohepcidin levels are followed up

\section{KARGER \\ Fax +4161306 1234 E-Mail karger@karger.ch} www.karger.com
(C) 2006 S. Karger AG, Base 0253-5068/06/0243-0309\$23.50/0

Accessible online at: www.karger.com/bpu
Prof. Francesco Locatelli

Department of Nephrology and Dialysis

A. Manzoni Hospital, Via Dell'Eremo 9-11

IT-23900 Lecco (Italy)

Tel. +39034148 9862, Fax +39034148 9860, E-Mail nefrologia@ospedale.lecco.it 
and analyzed against baseline hematocrit values, or experimental studies focusing on changes in plasma prohepcidin levels induced by rapidly changing hematocrit levels while controlling for potentially confounding covariates (above all, iron supplementation and inflammation) are the only means to gain insight into the pathophysiological link between hematocrit and prohepcidin levels that would allow us to draw definite conclusions concerning the existence of a cause-effect relationship (rather than a mere association) in patients undergoing chronic HD.
References

1 Hsu SP, Chiang CK, Chien CT, Hung KY: Plasma prohepdicin positively correlates with haematocrit in chronic haemodialysis patients. Blood Purif 2006;24:311-316.

2 Andrews NC: Anemia of inflammation: the cytokine-hepcidin link. J Clin Invest 2004;113: 1251-1253.

3 Nicolas G, Chauvet C, Viatte L, Danan JL, Bigard X, Devaux I, Beaumont C, Kahn A, Vaulont $\mathrm{S}$ : The gene encoding the iron regulatory peptide hepcidin is regulated by anemia, hypoxia, and inflammation. J Clin Invest 2002; 110:1037-1044. 